\title{
A Novel Method of Unsupervised Change Detection Using Multi-Temporal PolSAR Images
}

\author{
Wensong Liu ${ }^{1}$ (D), Jie Yang ${ }^{1, *}$, Jinqi Zhao ${ }^{1,2}$ (1) and Le Yang ${ }^{1}$ \\ 1 State Key Laboratory of Information Engineering in Surveying, Mapping and Remote Sensing, \\ Wuhan University, Wuhan 430079, China; liuwensongupc@163.com (W.L.); masurq@whu.edu.cn (J.Z.); \\ yangleupc@163.com (L.Y.) \\ 2 Huffington Department of Earth Sciences, Southern Methodist University, Dallas, TX 75275, USA \\ * Correspondence: yangj@whu.edu.cn; Tel.: +86-139-7151-2278
}

Received: 11 September 2017; Accepted: 4 November 2017; Published: 6 November 2017

\begin{abstract}
The existing unsupervised change detection methods using full-polarimetric synthetic aperture radar (PolSAR) do not use all the polarimetric information, and the results are subject to the influence of noise. In order to solve these problems, a novel automatic and unsupervised change detection approach based on multi-temporal full PolSAR images is presented in this paper. The proposed method integrates the advantages of the test statistic, generalized statistical region merging (GSRM), and generalized Gaussian mixture model (GMM) techniques. It involves three main steps: (1) the difference image (DI) is obtained by the likelihood-ratio parameter based on a test statistic; (2) the GSRM method is applied to the DI; and (3) the DI, after segmentation, is automatically analyzed by the generalized GMM to generate the change detection map. The generalized GMM is derived under a non-Gaussian assumption for modeling the distributions of the changed and unchanged classes, and automatically identifies the optimal number of components. The efficiency of the proposed method is demonstrated with multi-temporal PolSAR images acquired by Radarsat- 2 over the city of Wuhan in China. The experimental results show that the overall accuracy of the change detection results is improved and the false alarm rate reduced, when compared with some of the traditional change detection methods.
\end{abstract}

Keywords: PolSAR; unsupervised change detection; test statistic; generalized statistical region merging (GSRM); generalized Gaussian mixture model (GMM)

\section{Introduction}

In the field of remote sensing, change detection techniques are applied to recognize changes of the ground by analyzing remote sensing images taken at different times in the same geographical area $[1,2]$. Due to its all-time and all-weather mapping capability, which is independent of, for instance, cloud cover, night-time, and severe weather, the change detection of synthetic aperture radar (SAR) has played an important role in understanding the relationships and interactions between people and natural phenomena in recent years [3]. Change detection with SAR images has been used in applications such as disaster monitoring [4,5], ecological monitoring [6,7], regulatory policy development [8,9], and environmental impact assessment [10]. Meanwhile, full-polarimetric synthetic aperture radar (PolSAR) images contain both phase and amplitude information from radar returns transmitted in two different polarizations, and more scattering information can be obtained than with single-channel SAR images [11]. Therefore, many researchers have focused on change detection using multi-temporal PolSAR images [12-15].

Based on whether training data are accessed or not, change detection based on PolSAR images can be generally classified into two categories: unsupervised approaches and supervised approaches. 
Most of the supervised change detection approaches are dependent on the classification accuracy of the multi-temporal PolSAR images, and are liable to be affected by the significant cumulative error caused by the single remote sensing image classification during change detection [16,17]. Because of the impact of speckle noise, it is difficult to obtain high-precision classification results from multi-temporal PolSAR images, which limits the use of change detection based on PolSAR images. Unlike supervised change detection approaches, the unsupervised change detection methods implement change detection by directly comparing images acquired at different times, and these methods are widely used in change detection based on multi-temporal PolSAR images [18].

The traditional unsupervised methods based on PolSAR images usually take the pixel as the basic processing unit. The process of the state-of-the-art unsupervised change detection methods includes: (1) preprocessing; (2) generation of the difference image (DI); and (3) making a decision (thresholding algorithm or clustering algorithm) based on the analysis of the DI [19]. The preprocessing of multi-temporal PolSAR images mainly consists of radiometric calibration, speckle filtering, and image co-registration, which are all critical to the change detection. In particular, speckle filtering is usually carried out to suppress the speckle before the change detection of PolSAR images. Despite preprocessing, the result is still subject to the influence of noise and false alarms, which are caused by the pixel-based change detection method [20]. In generating the DI step, two preprocessed images of the same geographical area at different times are compared to generate the DI. There are many methods used to generate the DI using two co-registered images, including the ratio or log-ratio operator of SAR amplitudes or intensities [11,13,14], the hidden Markov chain model [16,17], and the Kullback-Leibler divergence method [18]. These methods are usually applied in multi-temporal single-channel SAR change detection. Unlike the above methods, test statistics can be applied not only to single-channel SAR data, but also to full PolSAR images [19]. Using multichannel PolSAR data (coherency $\mathrm{C}_{3}$ or covariance matrix $\mathrm{T}_{3}$ ) can obtain a more accurate DI [5]. In the step of change detection analysis, the change detection map can be obtained by a thresholding algorithm or a clustering algorithm. A number of different algorithms can be used to automatically make a decision, such as the $k$-means algorithm [21], the fuzzy c-means (FCM) algorithm [22], Otsu's thresholding method [23], Kapur's entropy algorithm [24], the Kittler and Illingworth (K\&I) algorithm [25], and two-dimensional entropic segmentation (TDES) [26]. However, most of the methods of making a decision are based on the condition of a Gaussian assumption for the probability density function (PDF) of the DI for modeling the distributions of the changed and unchanged classes. Fortunately, the Gaussian mixture model (GMM) is capable of better fitting the arbitrarily conditional densities of the classes in the DI $[27,28]$, but it is still difficult to select the optimal number of components for the GMM [29].

Above all, the existing pixel-based unsupervised change detection methods using full PolSAR images all show certain deficiencies, such as not taking advantage of the full-polarimetric information and being subject to the influence of noise and false alarms [17]. Meanwhile, the existing object-based unsupervised change detection methods using PolSAR images can suppress the influence of noise and improve the overall accuracy $(O A)$ of the change detection results [30], but they have difficulty in capturing the global property of the image. The results are also sensitive to segmentation, and this is difficult to accomplish due to the degradation of spatial details and fine structures [31]. To solve the above problems, a novel method of unsupervised change detection is proposed in this paper, integrating the respective advantages of the test statistic, generalized statistical region merging (GSRM), and generalized GMM techniques. The use of a test statistic is a good strategy for obtaining the DI from multi-temporal PolSAR images. We use the GSRM algorithm to separate the same parts of the DI, which helps us to choose the threshold by generalized GMM.

This paper is organized into five sections. In Section 2, the proposed change detection framework is described, and the methods of test statistic, GSRM, and generalized GMM techniques are introduced. Section 3 details the results of the proposed approach on multi-temporal PolSAR images from the city of Wuhan, China. Section 4 discusses the results of the case study. Finally, the conclusions are drawn in Section 5. 


\section{Theories and Methods}

\subsection{Test Statistic}

The full PolSAR system measures the amplitude and phase of each pixel's four basic polarization scattering echoes in four combinations of linear receive and transmit polarizations: horizontal-horizontal $(h h)$, horizontal-vertical $(h v)$, vertical-horizontal $(v h)$, and vertical-vertical (vv) [32]. Assuming that the target reciprocity condition is satisfied [33], the polarimetric information can be expressed by a complex vector:

$$
\Omega=\left[S_{h h}, \sqrt{2} S_{h v}, S_{v v}\right]^{T}
$$

where vector $\Omega$ follows a complex multivariate normal distribution. For multi-look processed PolSAR images, the covariance matrix follows a complex Wishart distribution, denoted as $X \in W\left(p, m, \Sigma_{X}\right)$. The covariance matrix $C$ is defined as:

$$
C=\left\langle\Omega \cdot \Omega^{* T}\right\rangle=\left\langle\left[\begin{array}{ccc}
\left|S_{h h}\right|^{2} & S_{h h} S_{h v}^{*} & S_{h h} S_{v v}^{*} \\
S_{h v} S_{h h}^{*} & \left|S_{h v}\right|^{2} & S_{h v} S_{v v}^{*} \\
S_{v v} S_{h h}^{*} & S_{v v} S_{h v}^{*} & \left|S_{v v}\right|^{2}
\end{array}\right]\right\rangle
$$

The frequency function of the covariance matrix $C$ is shown as follows:

$$
\begin{aligned}
& f(C)=\frac{1}{\Gamma_{n}(p)} \frac{1}{\left|\sum_{C}\right|^{n}}|C|^{n-p} \exp \left\{-\operatorname{tr}\left[\sum_{C}^{-1} C\right]\right\} \\
& \Gamma_{p}(n)=\pi^{p(p-1) / 2} \prod_{j=1}^{p} \Gamma(n-j+1)
\end{aligned}
$$

where $\operatorname{tr}(\cdot)$ is the trace operator, $n$ is the number of looks, $\Gamma_{p}(n)$ is a normalization factor, and $\sum_{C}=E\left[\left\langle\Omega \cdot \Omega^{* T}\right\rangle\right]$.

Let us consider two full PolSAR images $\left(X_{1}, X_{2}\right)$, which are independent, and both follow a complex Wishart distribution:

$$
\begin{aligned}
& X_{1} \in W\left(p, m, \sum_{X_{1}}\right) \\
& X_{2} \in W\left(p, n, \sum_{X_{2}}\right)
\end{aligned}
$$

where $p$ represents the dimension of $X_{1}, X_{2}$; and $m, n$ represent the number of looks of $X_{1}, X_{2}$, respectively. $\sum_{X_{1}}$ and $\sum_{X_{2}}$ represent the dispersion matrix of $X_{1}, X_{2}$. Their sum then has the same distribution $S \in W\left(p, m+n, \sum\right)$. The maximum likelihood estimation (MLE) of $\sum_{X_{1}}, \sum_{X_{2}}$, and $\sum_{X s}$ is shown as follows:

$$
\sum_{X_{1}}^{M L E}=\frac{1}{m} X_{1}, \sum_{X_{2}}^{M L E}=\frac{1}{n} X_{2} \text { and } \sum_{S}^{M L E}=\frac{1}{m+n}\left(X_{1}+X_{2}\right)
$$

According to test statistic theory [5], we make the hypothesis that the null hypothesis $H_{0}: \sum_{X_{1}}=\sum_{X_{2}}$ means that the two matrices are equal and there is a strong possibility of no change, and the alternative hypothesis $H_{1}: \sum_{X_{1}} \neq \sum_{X_{2}}$ means that the two matrices are different and there is a strong possibility of change [7].

Supposing that the test statistic based on MLE has a joint density $f\left(\sum_{X_{1}}, \sum_{X_{2}}, \theta\right)$, where $\theta$ is the set of parameters of the probability function that has generated the data. $H_{0}$ then states that $\theta \in H_{0}$, and the likelihood ratio of the test statistic is shown as follows:

$$
Q=\frac{\max _{\theta \in H_{0}} L(\theta)}{\max _{\theta \in \Omega} L(\theta)}=\frac{L\left(\sum_{S}\right)}{L_{X_{1}}\left(\sum_{X_{1}}\right) L_{X_{2}}\left(\sum_{X_{2}}\right)}
$$

where 


$$
\begin{gathered}
L_{X_{1}}\left(\sum_{X_{1}}\right) L_{X_{2}}\left(\sum_{X_{2}}\right)=\frac{1}{\Gamma_{p}(n) \Gamma_{p}(m)} \cdot\left|\sum_{X_{1}}\right|^{-n}\left|\sum_{X_{2}}\right|^{-m}\left|X_{1}\right|^{n-p}\left|X_{2}\right|^{m-p} \exp \left\{-\operatorname{tr}\left[\sum_{X_{1}}^{-1} X_{1}+\sum_{X_{2}}^{-1} X_{2}\right]\right\} \\
L\left(\sum_{S}\right)=\frac{1}{\Gamma_{p}(n) \Gamma_{p}(m)} \cdot\left|\sum\right|^{-(n+m)}\left|X_{1}\right|^{n-p}\left|X_{2}\right|^{m-p} \exp \left\{-\operatorname{tr}\left[\Sigma^{-1}\left(X_{1}+X_{2}\right)\right]\right\}
\end{gathered}
$$

This leads to the desired likelihood-ratio test statistic:

$$
Q=\frac{(n+m)^{p(n+m)}}{n^{p n} m^{p m}} \frac{\left|X_{1}\right|^{n}\left|Y_{1}\right|^{m}\left|X_{2}\right|^{n}\left|Y_{2}\right|^{m}}{\left|X_{1}+Y_{1}\right|^{n+m}\left|X_{2}+Y_{2}\right|^{n+m}}=\frac{(n+m)^{p(n+m)}}{n^{p n} m^{p m}} \frac{|X|^{n}|Y|^{m}}{|X+Y|^{n+m}}
$$

If $m=n$, Equation (7) is changed as follows:

$$
\operatorname{In} Q=n(2 q \operatorname{In} 2+\operatorname{In}|X|+\operatorname{In}|Y|-\operatorname{In}|X+Y|)
$$

where In $Q$ describes the similarity of the corresponding features, and its range is $(-\infty, 0]$. The closer In $Q$ is to 0 , the more similar the corresponding features, which means that change is less likely, and vice versa [30].

\subsection{Generalized Statistical Region Merging (GSRM)}

Speckle filtering is usually carried out to suppress the speckle before the change detection of PolSAR images. Despite the speckle filtering, the change detection result is still subject to the influence of noise and false alarms, which are caused by the pixel-based change detection method [20]. To solve the above problem, the GSRM algorithm [31] is applied to suppress the influence of noise.

The GSRM algorithm has been shown to perform well in solving significant noise corruption, and it does not depend on data distribution [31]. These advantages make GSRM suitable for the segmentation of the DI, which is characterized by speckle noise and different distributions of the various data types and spatial resolutions.

The GSRM algorithm belongs to the family of region growing and merging techniques. Accordingly, two essential components define a region merging algorithm: the merging predicate confirming whether adjacent regions are merged or not; and the order followed to test the merging of regions. Suppose $I$ is the DI that contains $|I|$ pixels, where $|\cdot|$ stands for the cardinal number. $Q_{R}$ is a parameter that enables the quantification of the statistical complexity of $I$.

\subsubsection{Merging Predicate}

From the Nock and Nielsen model, the following merging predicate is derived for the DI [31]:

$$
P\left(R, R^{\prime}\right)=\left\{\begin{array}{lc}
\text { true. } & \text { if }\left|\bar{R}^{\prime}-\bar{R}\right| \leq \sqrt{b^{2}(R)+b^{2}\left(R^{\prime}\right)} \\
\text { false. } & \text { otherwise }
\end{array}\right.
$$

where

$$
b(R)=g \sqrt{\frac{1}{2 Q_{R}|R|} \ln \frac{\left|R_{|R|}\right|}{\delta}}
$$

where $\bar{R}$ is the observed average for the DI in region $R ; R^{\prime}$ is the adjacent region of region $R ; \delta$ is the maximum probability when $P\left(R, R^{\prime}\right)=$ false, which is usually set as a very small value; $Q_{R}$ is a parameter which makes it possible to quantify the statistical complexity of the ideal segmentation, and also the statistical hardness of the task; $|R|$ is the pixel number in region $R ;|\cdot|$ stands for the cardinal number; and $R_{n}$ stands for the set of regions with $n$ pixels. 


\subsubsection{Merging Order}

For an observed image $I$, there are $N<2|I|$ pairs of adjacent pixels in 4-connectivity. $\mathrm{S}_{I}$ is the set of these pairs, and $f\left(p, p^{\prime}\right)$ is a real-valued function, with $p$ and $p^{\prime}$ a couple of adjacent pixels in $I$. Instead of stepwise optimization tactics, Nock and Nielsen proposed a pre-ordering strategy [32,33]. With this strategy, the GSRM algorithm can be described as follows: firstly, sort the pairs of $\mathrm{S}_{I}$ in increasing order of $f\left(p, p^{\prime}\right)$; and then traverse this order only once. For any current pair of pixels $\left(p, p^{\prime}\right) \in \mathrm{SI}$, if $R(p)=R\left(p^{\prime}\right)$, where $R(p)$ stands for the region to which $p$ belongs. Make the test $P\left(R(p), R^{\prime}\left(p^{\prime}\right)\right.$, and merge $R$ and $R^{\prime}$ if the result is true. The gradient function $f$ is defined as follows:

$$
f\left(p, p^{\prime}\right)=\left\|\frac{p-p^{\prime}}{p+p^{\prime}}\right\|_{1}
$$

Figure 1 shows the procedure of the GSRM algorithm.

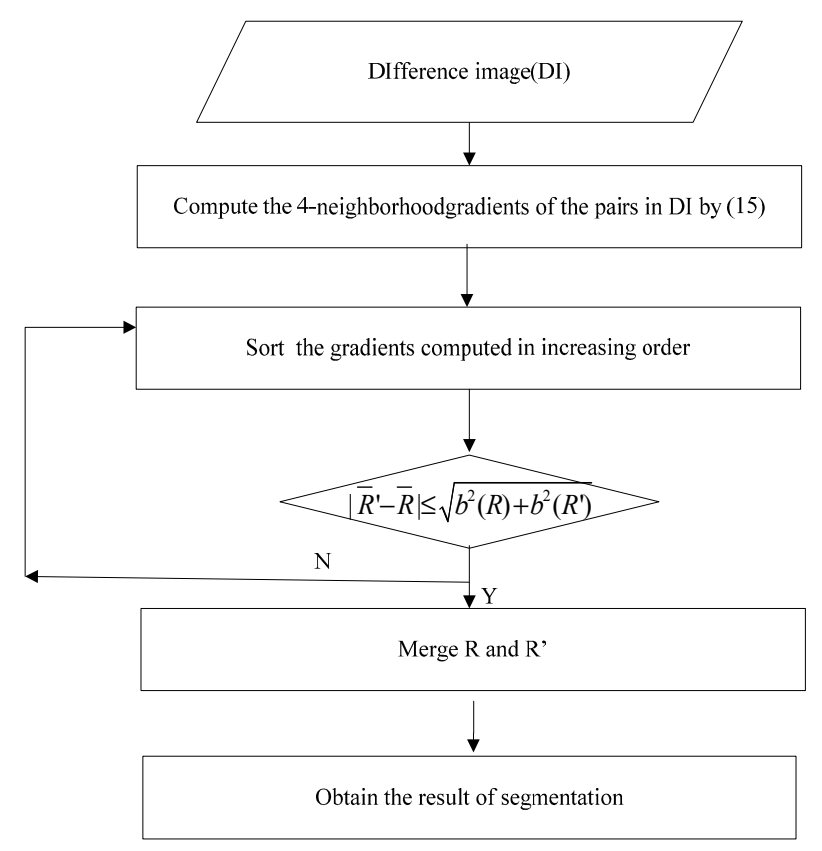

Figure 1. The procedure of the GSRM algorithm.

\subsection{The Generalized Gaussian Mixture Model (GMM) and Expectation Maximization (EM)}

The GMM [28] is an unsupervised statistical learning approach that is widely used in statistical pattern recognition. This parametric model is chosen because it is capable of better fitting the arbitrarily conditional densities of the classes in the DI after segmentation and it is suitable to fit an unknown data distribution of the DI after segmentation. The finite GMM with $k$ components can be written as:

$$
f(X \mid \theta)=\sum_{m=1}^{k} \alpha_{m} p\left(x \mid \theta_{m}\right)
$$

where $\alpha_{m}$ represent the mixing probabilities of the $m$-th component, which must be positive and sum to one; $\theta=\left\{\mu_{1}, \ldots, \mu_{m}, \sigma_{1}^{2}, \ldots, \sigma_{m}^{2}\right\} ; \mu_{k}$ are the means; $\sigma_{k}^{2}$ are the variances; and $p\left(x \mid \theta_{m}\right)$ is a normalized Gaussian with specified mean and variance.

In general, the data distribution of DI $p\left(x_{d}\right)$ after segmentation can be modeled as a weighted sum of two distributions representing the "changed" class and "unchanged" class data distributions [34], i.e.,

$$
p\left(x_{d}\right)=p\left(w_{u}\right) p\left(x_{d} \mid w_{u}\right)+p\left(w_{c}\right) p\left(x_{d} \mid w_{c}\right)
$$


where $p\left(x_{d} \mid w_{u}\right)$ and $p\left(x_{d} \mid w_{c}\right)$ are the a posteriori PDFs, and $p\left(w_{c}\right)$ and $p\left(w_{u}\right)$ are the a priori probabilities of classes $w_{u}$ and $w_{c}$, respectively. The data distribution $p\left(x_{d}\right)$ can also be modeled using the $k$-component GMM [35], i.e.,

$$
p\left(x_{d}\right)=\sum_{k=1}^{K} p(k) p\left(x_{d} \mid k\right)=\sum_{\forall \theta_{u} \in M_{u}} p\left(\theta_{u}\right) p\left(x \mid \theta_{u}\right)+\sum_{\forall \theta_{c} \in M_{c}} p\left(\theta_{c}\right) p\left(x \mid \theta_{c}\right)
$$

where $p(k)$ is the prior probability of the data point having been generated from component $n$ of the mixture, and $p\left(x_{d} \mid k\right)$ is the $n$-th component density modeled with a Gaussian density function. The data distribution modeled with Equation (15) can be separated into two distributions representing the data distributions of "changed" and "unchanged" classes.

In order to estimate $\theta$, it is usual to introduce the log-likelihood function defined as shown in Equation (15):

$$
\log p(x \mid \theta)=\log \prod_{i=1}^{n} p\left(x^{(\mathrm{i})} \mid \theta\right)=\sum_{i=1}^{n} \log \sum_{m=1}^{k} \alpha_{m} p\left(x^{(\mathrm{i})} \mid \theta_{m}\right)
$$

The MLE is:

$$
\hat{\theta}_{M L}=\underset{\theta}{\operatorname{argmax}}\{\log p(x \mid \theta)\}
$$

However, $x=\left\{x^{(1)}, \ldots, x^{(n)}\right\}$ are incomplete data, and Equation (16) cannot be solved using a derivative of 0 . It is worth noting that the expectation maximization (EM) algorithm [36] is a special case of the GMM algorithm when the feature vector for each pixel constructed with no contextual information is used for the GMM representation [37].

The EM algorithm is an iterative method used to find the maximum likelihood or the maximum a posteriori estimates of the components in the GMM [36]. The EM iteration alternates between performing an expectation (E) step and a maximization (M) step. The E-step creates a function for the expectation of the log-likelihood evaluated using the current estimate for the parameters:

$$
Q(\theta, \hat{\theta}(t)) \equiv \mathrm{E}[\log p(X, \gamma \mid \theta) \mid X, \hat{\theta}(\mathrm{t})]=\log p(X, \hat{\gamma} \mid \theta)
$$

where

$$
\hat{\gamma}_{i m} \equiv E\left[\gamma_{i m} \mid x, \hat{\theta}(\mathbf{t})\right]=\frac{\hat{\alpha}_{m}(\mathrm{t}) p\left(x_{i} \mid \hat{\theta}_{m}(\mathrm{t})\right)}{\sum_{j=1}^{k} \hat{\alpha}_{j}(\mathrm{t}) p\left(x_{i} \mid \hat{\theta}_{j}(\mathrm{t})\right)}
$$

The M-step computes the parameters maximizing the expected log-likelihood found in the E-step.

$$
\frac{\partial(Q(\theta, \hat{\theta}(\mathrm{t})))}{\partial \theta}=0
$$

We update the parameter estimates according to Equation (20):

$$
\begin{gathered}
\alpha_{i}=\sum_{i=1}^{n} \hat{\gamma}_{i m} / n \\
\mu_{i}=\sum_{i=1}^{n} \hat{\gamma}_{i m} x_{i} / \sum_{i=1}^{n} \hat{\gamma}_{i m} \\
\sigma_{i}^{2}=\sum_{i=1}^{n} \hat{\gamma}_{i m}\left(x_{i}-\mu_{i}\right)^{T}\left(x_{i}-\mu_{i}\right) / d \sum_{i=1}^{n} \hat{\gamma}_{i m}
\end{gathered}
$$


The EM algorithm of the criterion of convergence is defined as shown in Equation (23):

$$
\|\theta-\hat{\theta}(t)\|<\delta
$$

For a certain class of traditional GMM algorithm, there is an important parameter commonly referred to as $K$ that specifies the number of components to detect $[27,28]$. However, the correct choice of $K$ is often ambiguous. Furthermore, it is essential to find the optimal number of components $K$, for which the elbow method [38] is introduced in this paper.

The elbow method is a method of interpretation and validation of the consistency within cluster analysis that is designed to help find the appropriate number of clusters in a dataset. It looks at the percentage of variance explained as a function of the number of clusters, i.e., one should choose a number of clusters so that adding another cluster does not give a much better modeling of the data. However, this "elbow" cannot always be unambiguously identified [38]. $K$ is the cluster number, and it corresponds to $90 \%$ of the variance, explained as the ratio of the between-group variance to the total variance, which is also known as an F-test [39]. The elbow method is shown as follows:

$$
F=\frac{\text { between_group } \operatorname{variance}\left(F_{B}\right)}{\text { total } \operatorname{variance}\left(F_{T}\right)}
$$

where

$$
\begin{gathered}
F_{B}=\sum_{i=1}^{K} n_{i}\left(\overline{Y_{i}}-\bar{Y}\right)^{2} /(K-1) \\
F_{T}=\sum_{i=1}^{K} \sum_{j=1}^{n_{i}}\left(Y_{i j}-\overline{Y_{i}}\right)^{2} /(N-K)
\end{gathered}
$$

where $\overline{Y_{i}}$ denotes the sample mean in the $i$-th group, $n_{i}$ is the number of observations in the $i$-th group, $\bar{Y}$ denotes the overall mean of the data, and $K$ denotes the number of groups. $Y_{i j}$ is the $j$-th observation in the $i$-th out of $K$ groups, and $N$ is the overall sample size.

Figure 2 shows the procedure of the GMM and EM algorithms.

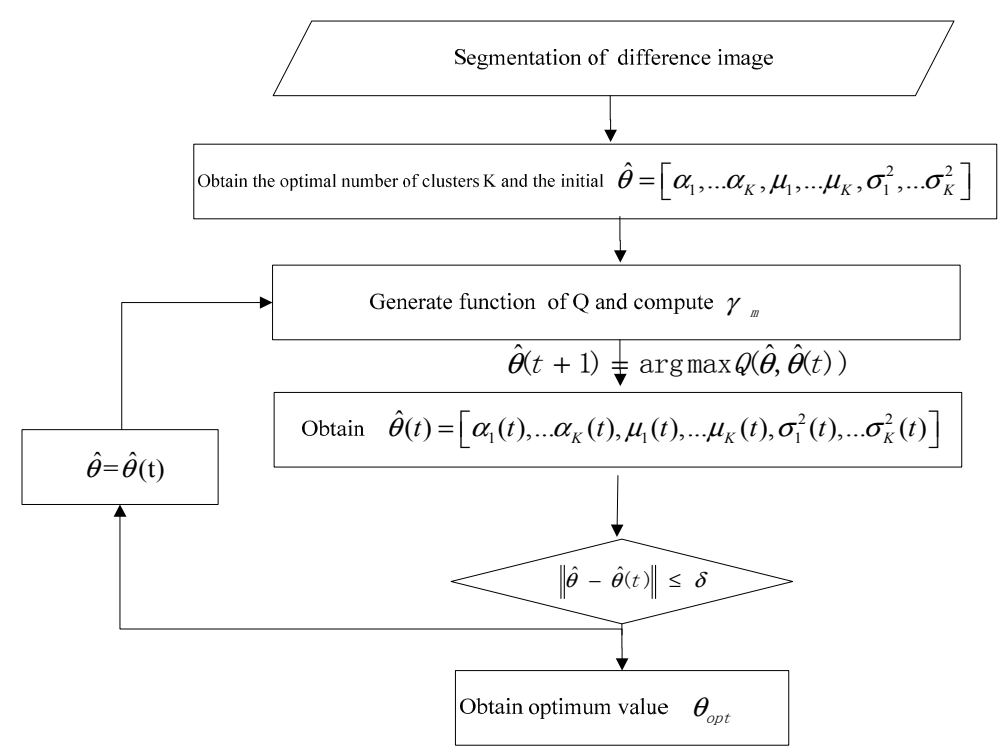

Figure 2. The procedure of the GMM and EM algorithms. 


\subsection{The Proposed Method of TS_srm_GMM}

The basic process flow of the proposed method consists of: (1) data preprocessing, which consists of speckle filtering and image co-registration; (2) generation of the DI using the test statistic; (3) segmentation of the DI by GSRM; and (4) obtaining the PDF of the changed and unchanged classes using generalized GMM and making a decision based on Equation (27):

$$
C D(i, j)=\left\{\begin{array}{l}
1, p\left(w_{u}\right) p\left(x \mid w_{u}\right)<p\left(w_{c}\right) p\left(x \mid w_{c}\right) \\
0, \text { otherwise }
\end{array}\right.
$$

where " 1 " represents changed classes and " 0 " represents unchanged classes. The process flow of the proposed method is shown in Figure 3.

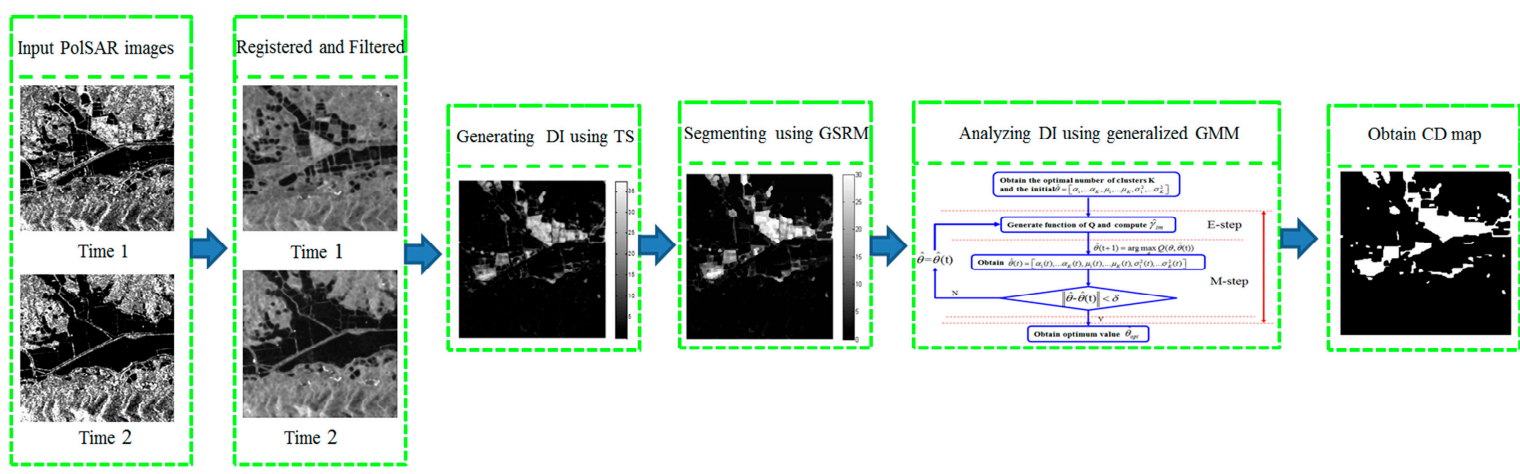

Figure 3. The process flow of the proposed method.

Firstly, speckle noise is removed by a $7 \times 7$ refined Lee filter for the two registered full PolSAR images. The DI is obtained by the likelihood-ratio parameter, which is built using coherency matrix C3 or covariance matrix T3 of the full PolSAR images based on the test statistic. To suppress the influence of speckle noise in the DI, the GSRM algorithm is applied to segment the DI. The data distribution of the DI after segmentation computed from satellite images of the same scene acquired at different times is modeled using the generalized GMM. It also becomes possible to automatically extract change information based on Equation (27). Finally, the precision of the change detection result is evaluated.

\subsection{Evaluation Criterion}

Quantitative evaluation is important to determine the result of change detection. When the ground reference is available, quantitative evaluation can be performed [40,41]. In order to verify the performance of the proposed method, we calculated the false alarm $(F A)$ rate, omission factor $(O F)$ rate, overall accuracy $(O A)$, and Kappa coefficient [42] of the experimental results. These indicators are calculated as follows:

$$
\left\{\begin{array}{l}
F A=\frac{F P}{N_{u}} \\
O F=\frac{F N}{N_{S}} \\
O A=\frac{T P+T N}{N} \\
K a p p a=\frac{O A-P e}{1-P e} \\
P e=\frac{(T P+F N)(T P+F P)+(F P+T N)(F N+T N)}{N^{2}}
\end{array}\right.
$$

where $F P$ means the number of unchanged points incorrectly detected as changed; $F N$ means the number of changed points incorrectly detected as unchanged; TP means the number of changed points correctly detected; $T N$ means the number of unchanged points correctly detected; $N_{u}$ and $N_{c}$ are the number of unchanged points and changed points of the ground-truth change map; $N$ is the sum of $N_{u}$ and $N_{c}$, respectively. 


\section{Experiments and Results}

\subsection{Study Area and Data}

The city of Wuhan is the capital of Hubei province in China. It is situated between latitude $29^{\circ} 58^{\prime}-31^{\circ} 22^{\prime} \mathrm{N}$ and longitude $113^{\circ} 41^{\prime}-115^{\circ} 05^{\prime} \mathrm{E}$. Wuhan lies in the eastern part of the Jianghan Plain, and is located at the confluence of the Hanshui and Yangtze rivers, along the middle reaches of the latter. Most of the area is plain, with scattered hills and a great number of lakes and pools, as shown in Figure 4. The climate is humid subtropical, with abundant rainfall and four distinct seasons. In July 2016, 50-year return period rainfall occurred, and the rainfall was significantly higher than the average annual precipitation. According to reports, the city of Wuhan was badly affected by the continuous heavy rain, and some areas showed dramatic change, especially YanDong Lake and LiangZi Lake. Optical sensors are of limited use in such severe weather. In order to detect the flooded regions, multi-temporal PolSAR images were acquired, allowing a rapid emergency response [42].

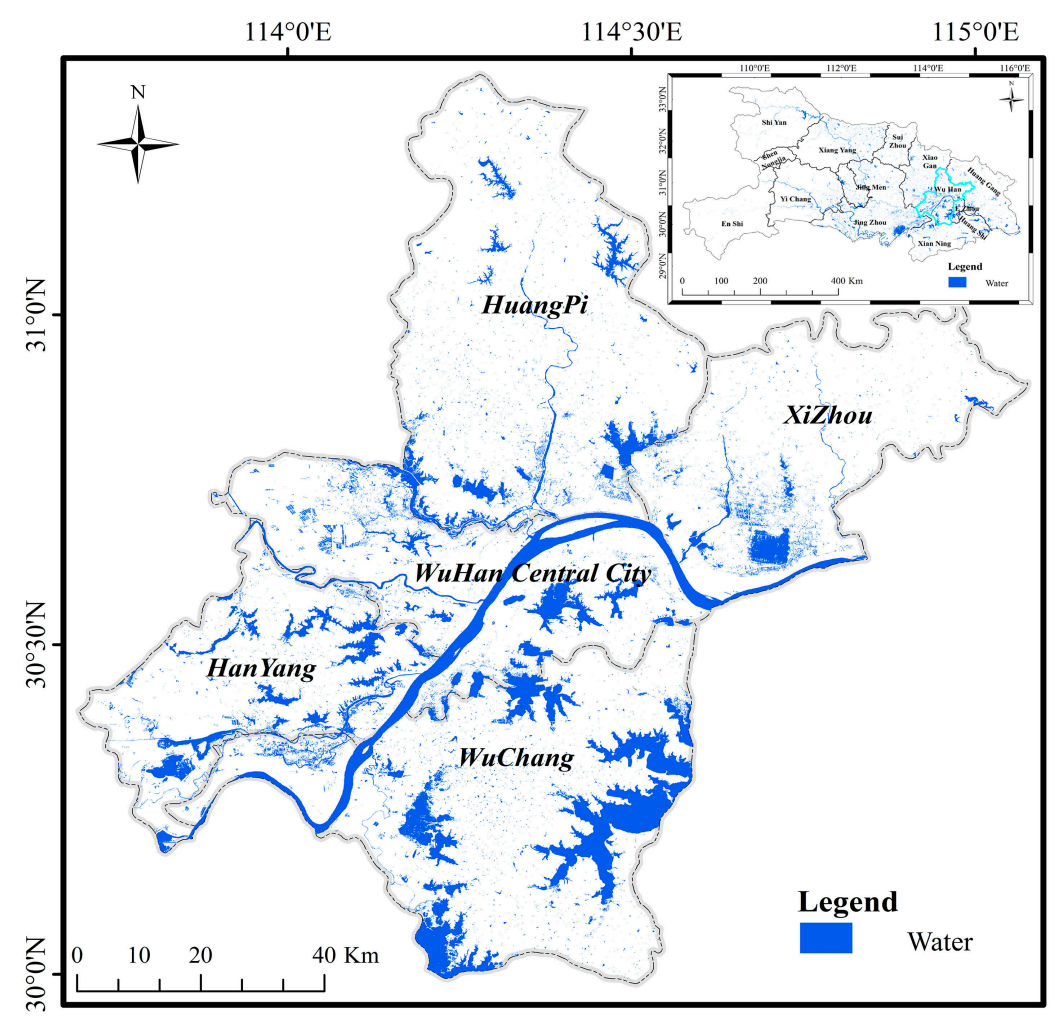

Figure 4. Location of the study area.

In order to assess the effectiveness of the change detection algorithms for the analysis of the DI, multi-temporal Radarsat-2 data of the flood disaster in Wuhan in 2016 are considered in this paper. The two C-band quad-polarimetric Radarsat-2 images (single look complex) are composed of two images which are regions from two SAR images acquired by Radarsat-2 over an area near the city of Wuhan, China, on 25 June 2015 and 6 July 2016, respectively. The Pauli-RGB images $\left(\left|S_{h h}-S_{v v}\right|\right.$ for red, $\left|S_{h v}\right|$ for green, and $\left|S_{h h}+S_{v v}\right|$ for blue) are shown in Figure 5 (YanDong Lake) and Figure 6 (LiangZi Lake). The ground reference of the changed areas was obtained by visual interpretation of the PolSAR data and corresponding optical images. 


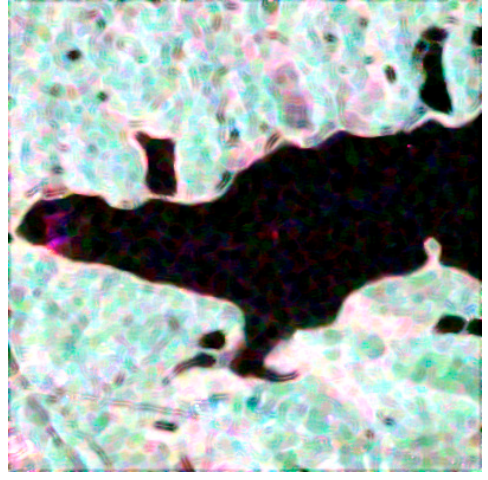

(a)

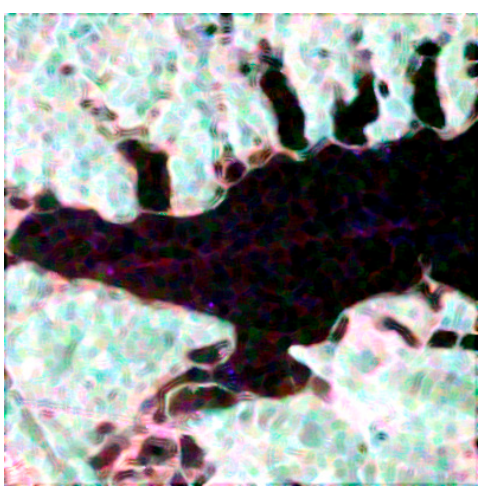

(b)

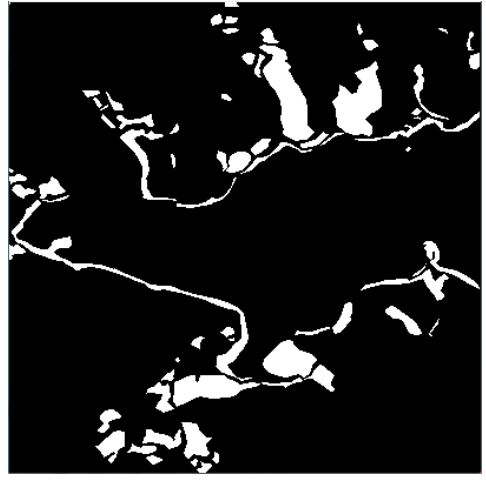

(c)

Figure 5. The Pauli-RGB images of YanDong Lake after preprocessing on (a) 25 June 2015, and (b) 6 July 2016. (c) Ground reference.

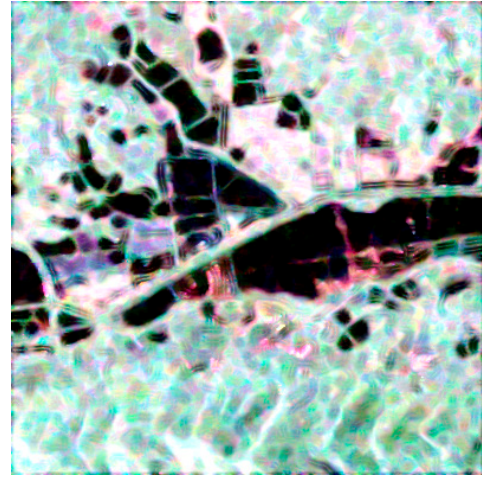

(a)

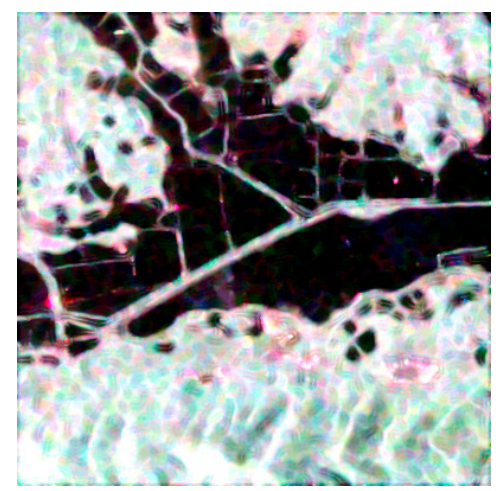

(b)

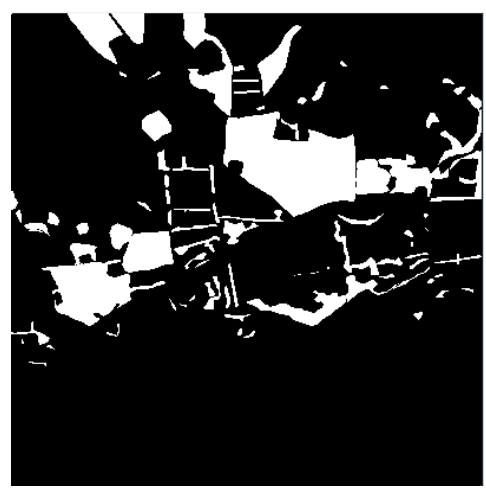

(c)

Figure 6. The Pauli-RGB images of Liangzi Lake after preprocessing on (a) 25 June 2015, and (b) 6 July 2016. (c) Ground reference.

The multi-temporal PolSAR images of YanDong Lake shown in Figure 5a,b were acquired on 25 June 2015, and 6 July 2016, respectively. The image size is $400 \times 400$ pixels. The area of YanDong Lake includes some grassland, city, and water bodies. The main changes occurred in the water bodies, because of the heavy rain.

The multi-temporal PolSAR images of LiangZi Lake shown in Figure 6a,b were acquired on 25 June 2015, and 6 July 2016, respectively. The image size is $300 \times 500$ pixels. The ground reference of the changed areas was obtained by visual interpretation of the PolSAR data and corresponding optical images. The area of LiangZi Lake includes some grassland, city, and water bodies. The main changes again occurred in the water bodies.

\subsection{Implementation of the Change Detection Algorithms}

The DI for YanDong Lake based on the test statistic uses all the information of polarimetric SAR. The DI is shown in Figure 7a and the histogram of the DI is shown in Figure 7b.

In Figure 7a, the DI for YanDong Lake based on the test statistic can accurately detect the difference in information among the multi-temporal PolSAR images, but it still contains speckle noise. Figure $7 \mathrm{~b}$ shows the histogram of the DI based on pixels, and we can see that the DI still contains many isolated outliers. Moreover, the probability distribution of the DI is far from a Gaussian distribution. To suppress the influence of noise, the GSRM algorithm was applied to the DI of YanDong Lake, and the result is shown in Figure 8. 


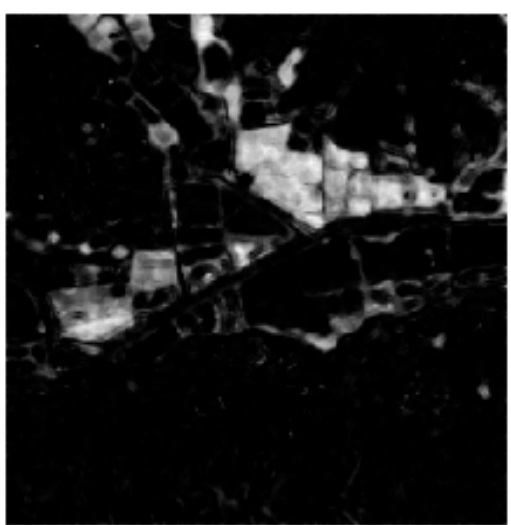

(a)

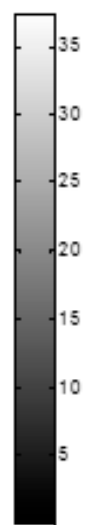

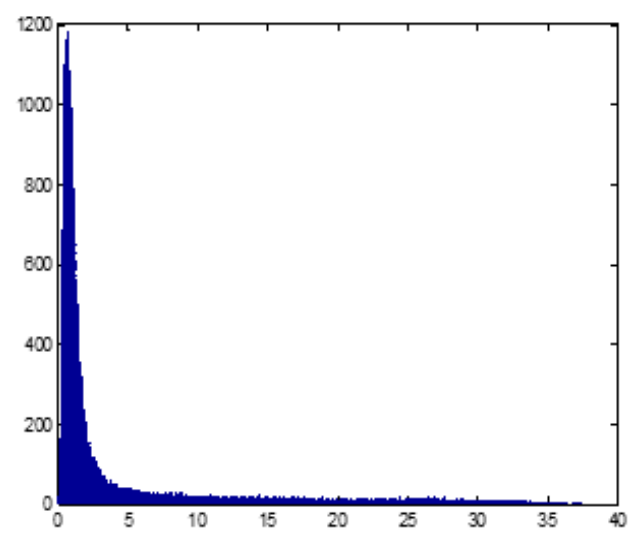

(b)

Figure 7. (a) The DI based on pixels; (b) Histogram of the DI based on pixels.

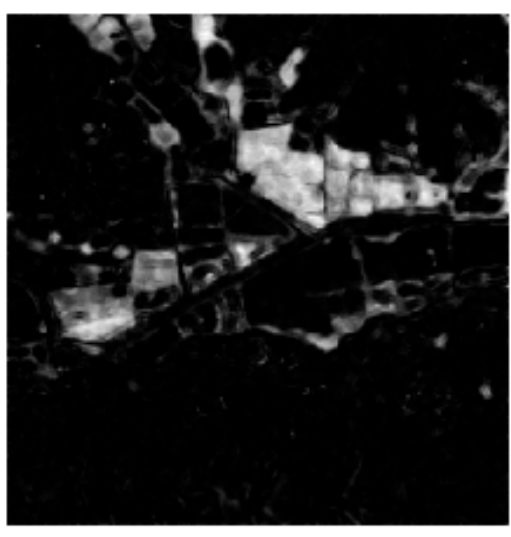

(a)

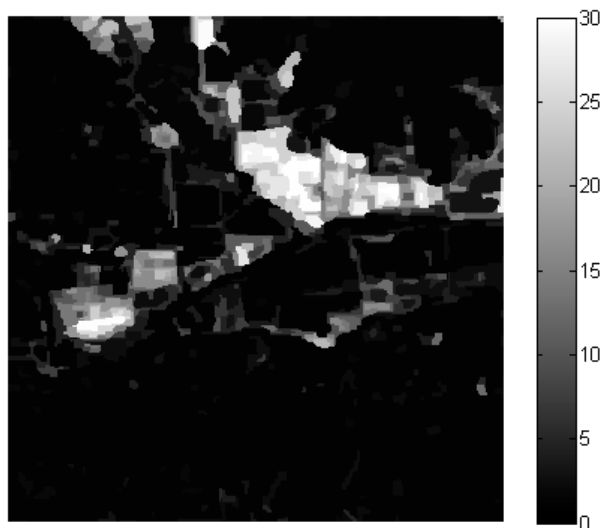

(b)

Figure 8. (a) The DI based on pixels; (b) The result of GSRM.

The DI for YanDong Lake was segmented based on GSRM, which is shown in Figure 9a. It can be seen that the GSRM technique produces smooth results and suppresses the influence of noise in the DI. The histogram is shown in Figure 9b, where many isolated outliers can be picked out. Moreover, the probability distribution of the DI is under a non-Gaussian distribution.

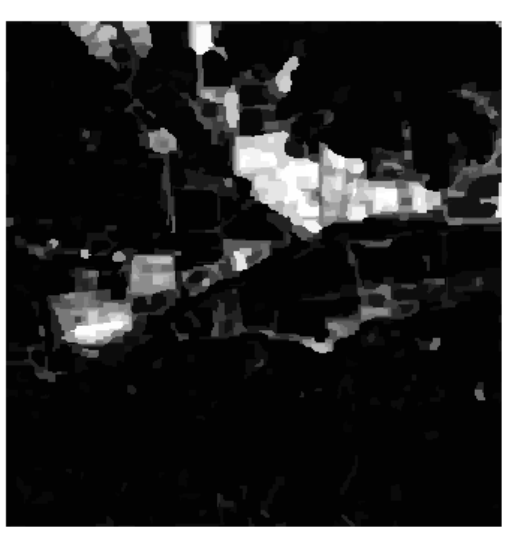

(a)
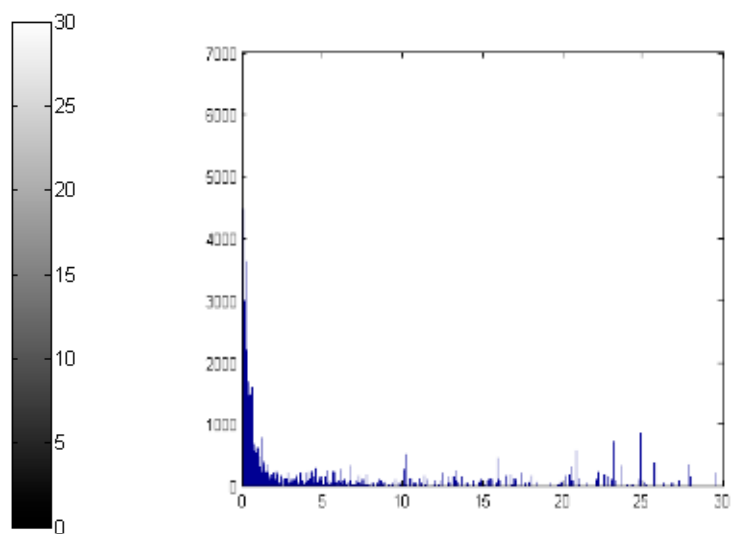

(b)

Figure 9. (a) The DI after GSRM; (b) Histogram of the DI after GSRM. 


\subsection{Results}

In order to assess the effectiveness of the proposed approach, contrast experiments were designed. The contrast experiments included unsupervised change detection based on pixels (test statistic and GMM, named TS_GMM_pixel), based on objects (test statistic and GMM, named TS_GMM_object) and the approach proposed in this paper, including Log_ratio and GMM (named Log_srm_GMM), test statistic and K\&I (named TS_srm_KI), test statistic and TDES (named TS_srm_TDES), test statistic and $k$-means (named TS_srm_kmeans), test statistic and FCM (named TS_srm_FCM), and test statistic and GMM (named TS_srm_GMM). The results for the multi-temporal PolSAR images of YanDong Lake based on the eight methods are shown in Figure 10. The change detection map shows change (white) and no-change (black) information.

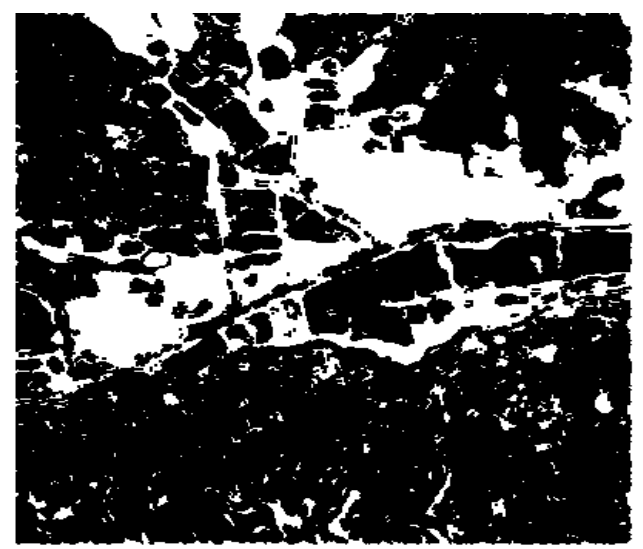

(a) TS_GMM_pixel $(k=19)$

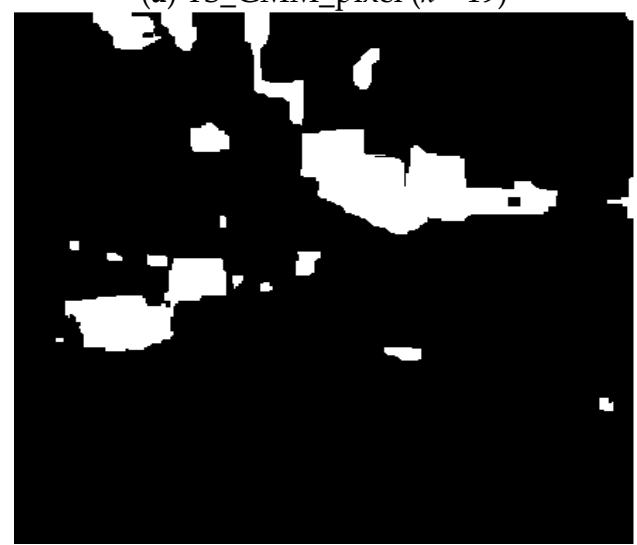

(c) Log_srm_GMM $(k=24)$

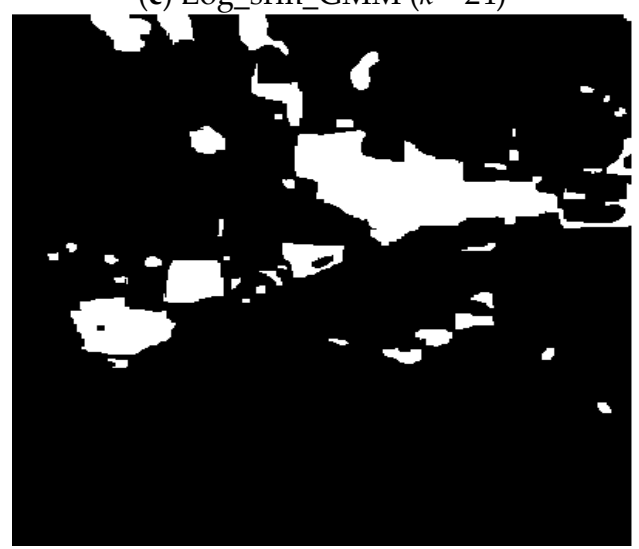

(e) TS_srm_TDES

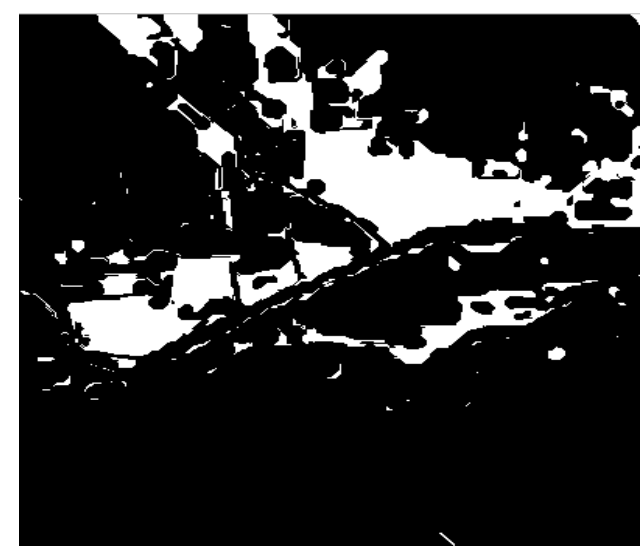

(b) TS_GMM_object $(k=32)$

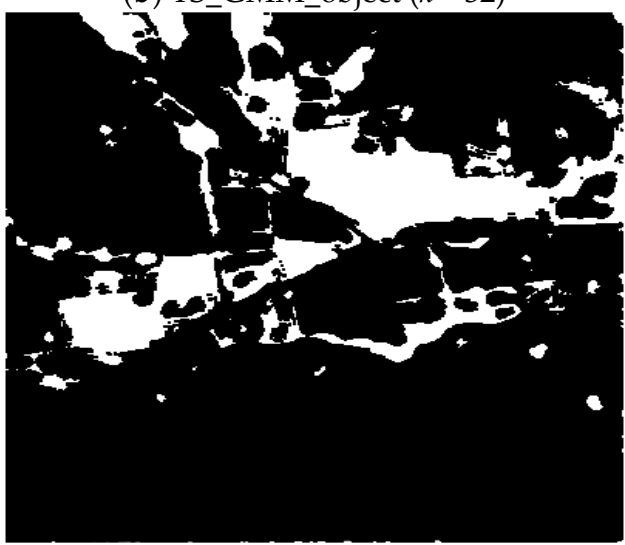

(d) TS_srm_KI

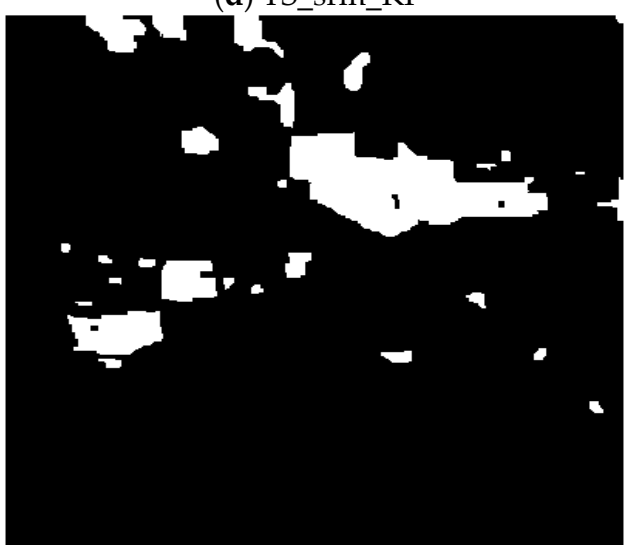

(f) TS_srm_kmeans

Figure 10. Cont. 


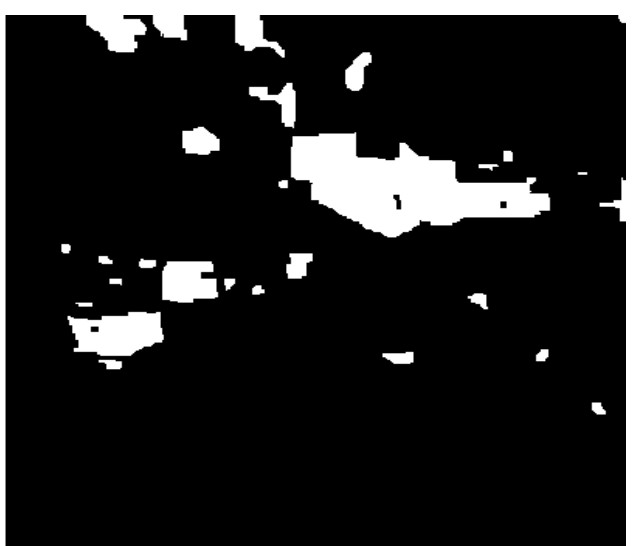

(g) TS_srm_FCM

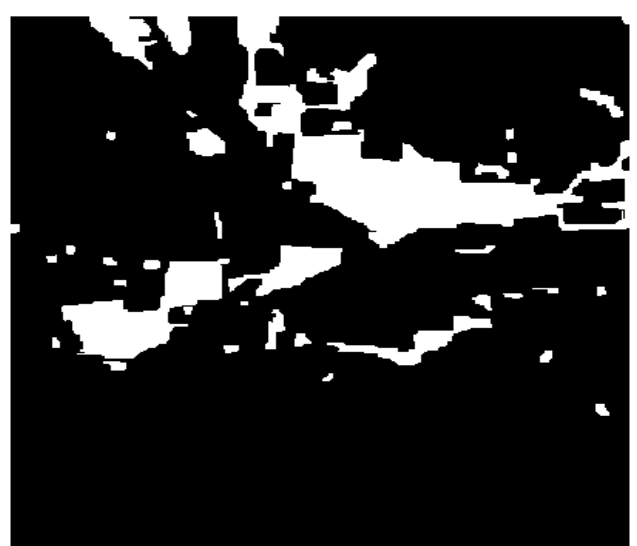

(h) TS_srm_GMM $(k=27)$

Figure 10. Change detection results of the different algorithms over YanDong Lake.

The change detection results of the different algorithms over YanDong Lake are shown in Figure 10. Figure 10a shows the change detection result using the test statistic and generalized GMM algorithm based on pixels, which can detect the spatial details and fine structures of the change, but it causes a noisy change detection map, with holes in connected regions and jagged boundaries. Figure 10b shows the change detection result using the test statistic and generalized GMM algorithm based on objects, which can suppress the speckle noise; however, this is accompanied with degradation of the spatial details and it is more time-consuming. Figure 10c shows the change detection result using the Log_ratio algorithm and generalized GMM algorithm based on the approach proposed in this paper, which can better suppress the speckle noise; however, it does have a higher OF. Figure 10d-g show the change detection results using the test statistic and the traditional algorithms (KI, TDES's, $k$-means, FCM) based on the approach proposed in this paper, which can better suppress the speckle noise; however, they contain more fallout information. Figure 10h shows the change detection result using the test statistic and generalized GMM algorithm based on the approach proposed in this paper, which not only better suppresses the speckle noise, but also has a higher $O A$ than the results of the traditional algorithms.

In order to compare each of the different results with ground reference over YanDong Lake, we overlaid each of the different results with ground reference which improved the readability of Figure 10 and are shown in Figure 11. Where the green represents the match of proposed result in comparison to the ground reference (overall accuracy), the red represents the underestimation of the proposed methodology in comparison with the ground reference (omission factor $(O F)$ rate) and the blue represents the overestimation of the proposed methodology in comparison with the ground reference (false alarm $(F A)$ rate).

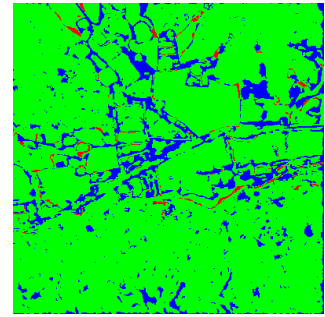

(a) TS_GMM_pixel

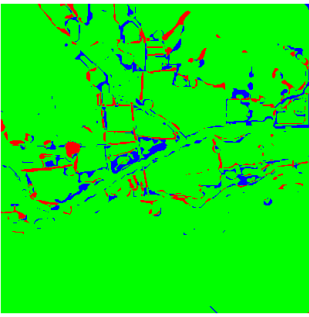

(b) TS_GMM_object

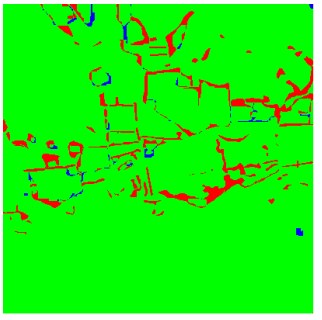

(c) Log_srm_GMM

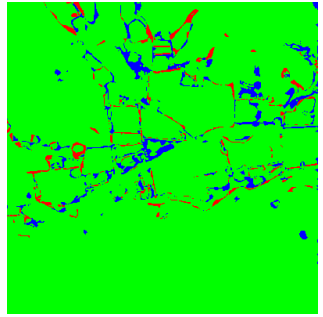

(d) TS_srm_KI

Figure 11. Cont. 


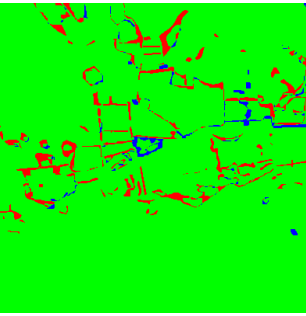

(e) TS_srm_TDES

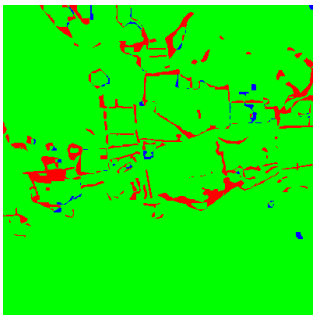

(f) TS_srm_kmeans

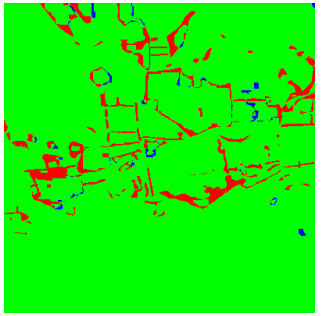

(g) TS_srm_FCM

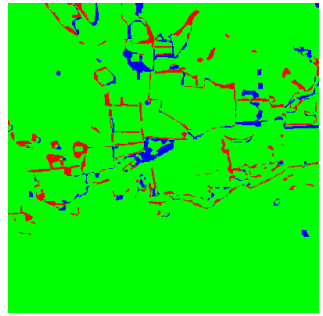

(h) TS_srm_GMM

Figure 11. The qualitative comparisons change detection results of the different algorithms over YanDong Lake.

Several conclusions can be obtained from Figure 11. The TS_srm_GMM approach shows a better performance than the other approaches and it obtains a lower $F A$ than other approaches (such as TS_GMM_pixel, TS_GMM_object, Log_srm_GMM and TS_srm_KI). It also obtains a lower OF than other approaches (such as Log_srm_GMM, TS_srm_KI, TS_srm_kmeans and TS_srm_FCM) in qualitative comparisons over YanDong Lake.

The quantitative comparisons of the eight detection schemes shown in Table 1 indicate that the proposed approach shows a better performance than the other approaches. The proposed approach achieves the best results on two indicators (OA (94.47\%) and Kappa (0.75)), and it obtains a lower $F A(1.68 \%)$ than the pixel-based (2.95\%) and object-based (3.63\%) approaches. Moreover, the $O A$ of the change detection results is improved $(0.83 \%)$ and the false alarm rate reduced $(1.27 \%)$ using the proposed method, compared with some of the traditional methods. Both the qualitative and quantitative analyses demonstrate that the proposed method is more effective and shows a significant improvement over the traditional methods.

In order to further assess the effectiveness and feasibility of the proposed approach, the multi-temporal PolSAR images from LiangZi Lake were used to test the proposed approach. The change detection results of the different algorithms over LiangZi Lake are shown in Figure 11.

Figure 12a shows the change detection result using the test statistic and generalized GMM algorithm based on pixels, which can detect the spatial details and fine structures of the change, but it causes a noisy change detection map, with holes in connected regions and jagged boundaries. Figure $12 \mathrm{~b}$ shows the change detection result using the test statistic and generalized GMM algorithm based on objects, which can suppress the speckle noise; however, it is accompanied with degradation of the spatial details and it is more time-consuming. Figure 12c shows the change detection result using the Log_ratio algorithm and generalized GMM algorithm based on the approach proposed in this paper, which can also suppress the speckle noise; however, it does have a higher OF. Figure $12 \mathrm{~d}-\mathrm{g}$ show the change detection result using the test statistic and the traditional algorithms (KI, TDES's, $k$-means, FCM) based on the approach proposed in this paper, which can better suppress the speckle noise; however, they contain more fallout information. Figure $12 \mathrm{~h}$ shows the change detection result using the test statistic and generalized GMM algorithm based on the approach proposed in this paper, which not only better suppresses the speckle noise, but also has a higher $O A$ than the traditional algorithms.

Table 1. Performance evaluation of the change detection over YanDong Lake.

\begin{tabular}{ccccc}
\hline Method & $\boldsymbol{O A}$ & $\boldsymbol{F A}$ & $\boldsymbol{O F}$ & Kappa \\
\hline TS_GMM_pixel & $93.64 \%$ & $2.95 \%$ & $3.41 \%$ & 0.72 \\
TS_GMM_object & $93.55 \%$ & $3.63 \%$ & $2.82 \%$ & 0.73 \\
Log_srm_GMM & $92.80 \%$ & $2.13 \%$ & $5.07 \%$ & 0.68 \\
TS_srm_KI & $93.39 \%$ & $2.47 \%$ & $4.13 \%$ & 0.70 \\
TS_srm_TDES & $93.99 \%$ & $1.31 \%$ & $4.70 \%$ & 0.72 \\
TS_srm_kmeans & $93.32 \%$ & $0.88 \%$ & $6.24 \%$ & 0.70 \\
TS_srm_FCM & $93.92 \%$ & $0.75 \%$ & $5.31 \%$ & 0.69 \\
TS_srm_GMM & $94.47 \%$ & $1.68 \%$ & $3.84 \%$ & 0.75 \\
\hline
\end{tabular}




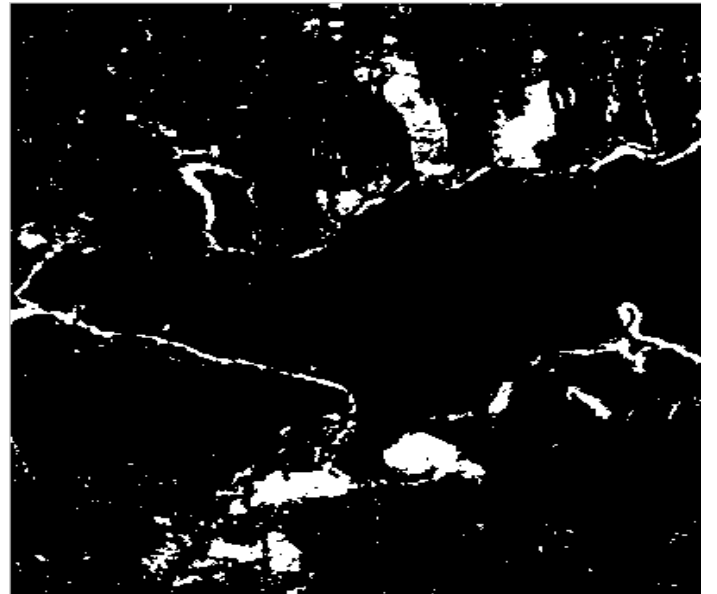

(a) TS_GMM_pixel $(k=21)$

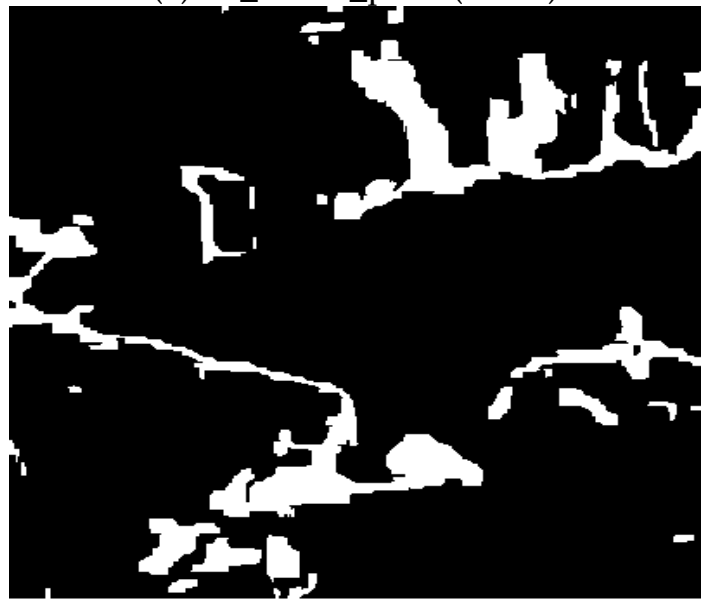

(c) Log_srm_GMM $(k=18)$

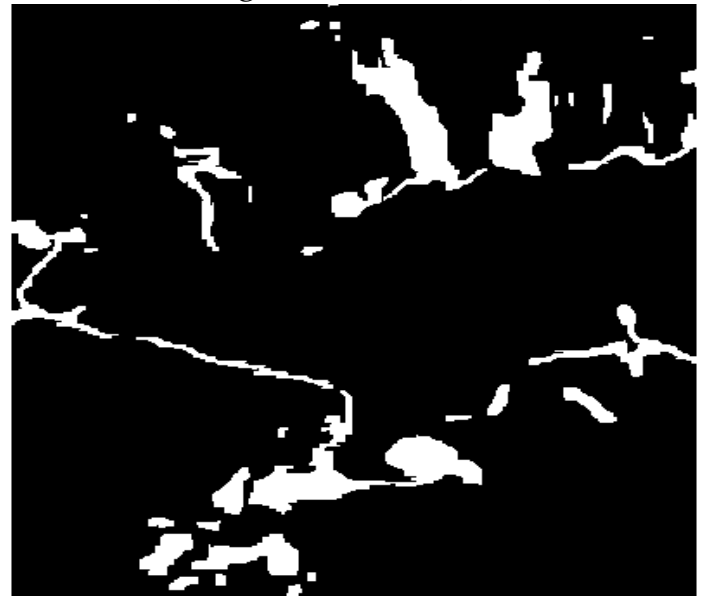

(e) TS_srm_TDES

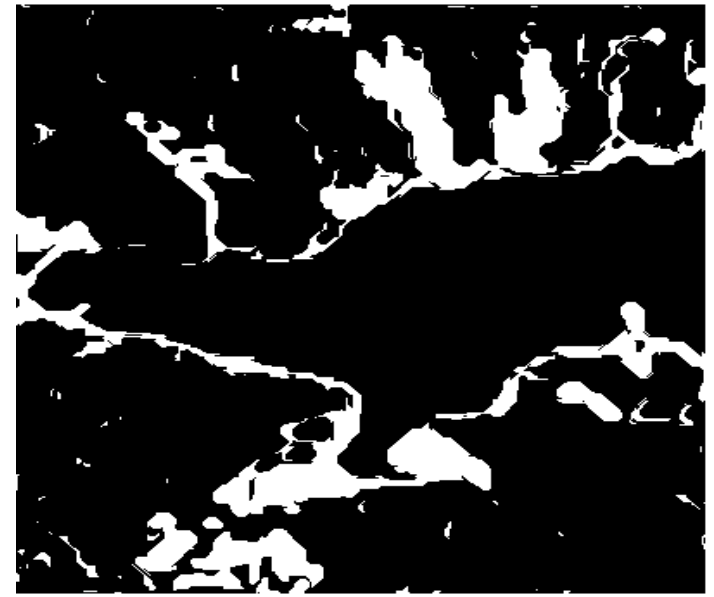

(b) TS_GMM_object $(k=33)$

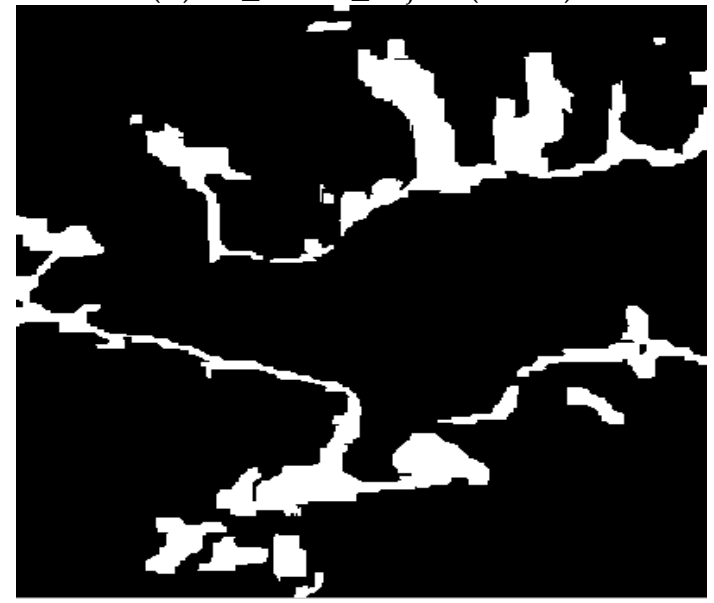

(d) TS_srm_KI

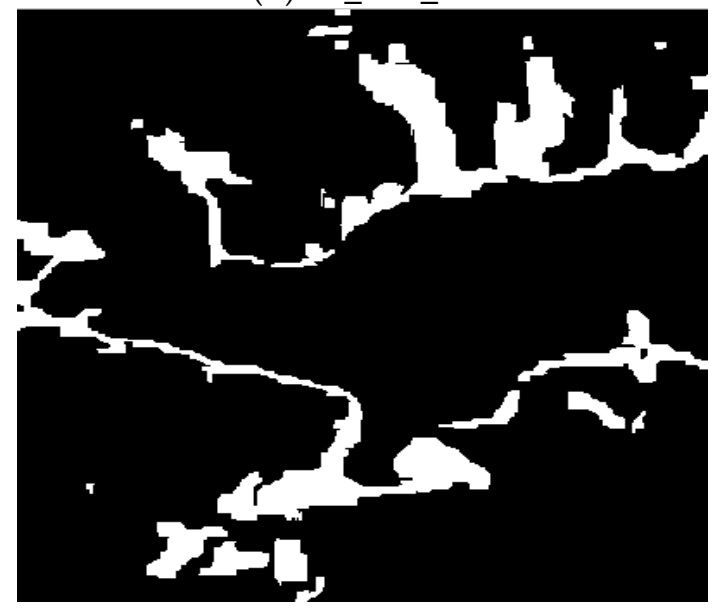

(f) TS_srm_kmeans

Figure 12. Cont. 


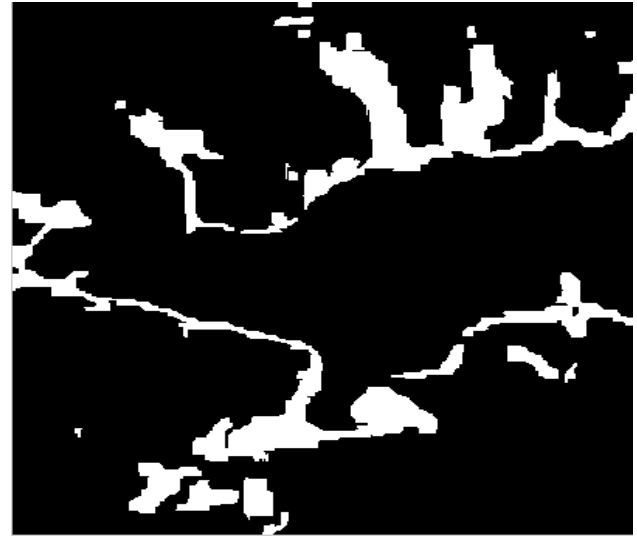

(g) TS_srm_FCM

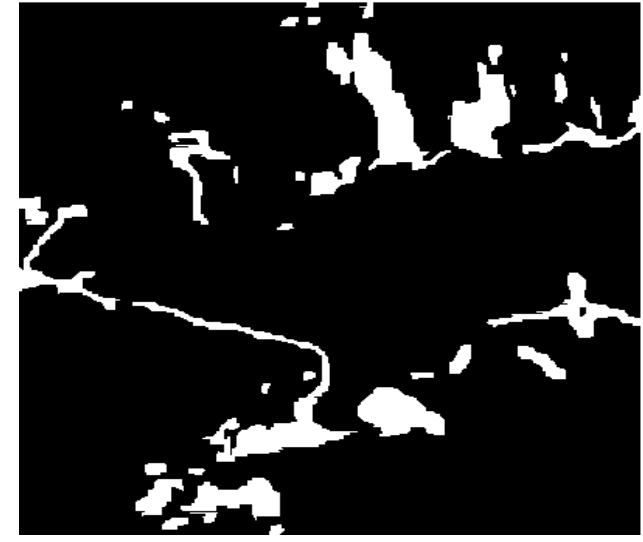

(h) TS_srm_GMM $(k=25)$

Figure 12. Change detection results of the different algorithms over LiangZi Lake.

Analogously, Figure 13 shows that the qualitative comparisons change detection results of the different algorithms with ground reference over LiangZi Lake.

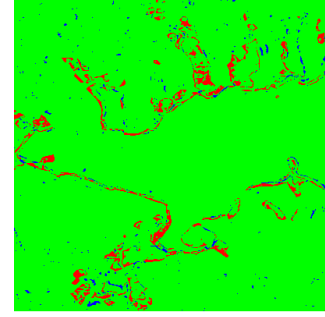

(a) TS_GMM_pixel

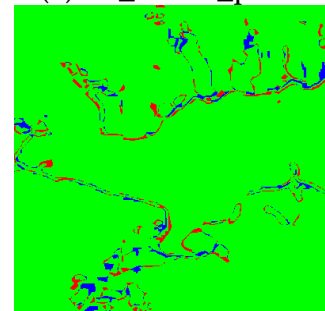

(e) TS_srm_TDES

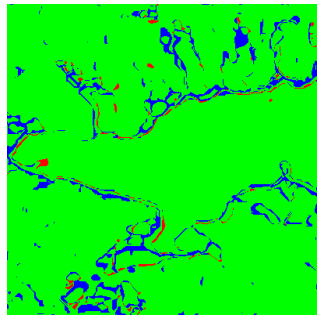

(b) TS_GMM_object

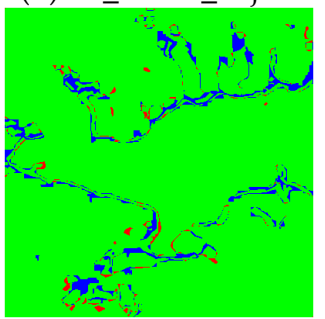

(f) TS_srm_kmeans

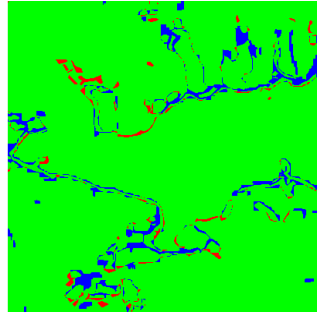

(c) Log_srm_GMM

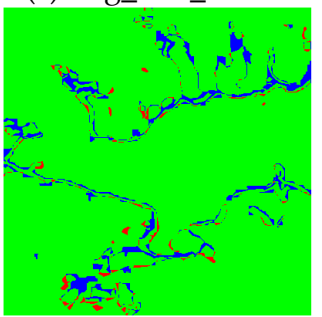

(g) TS_srm_FCM

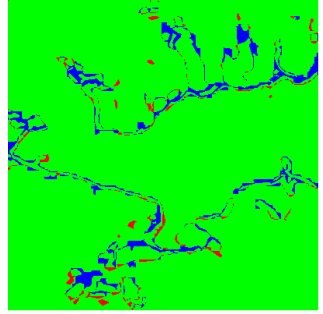

(d) TS_srm_KI

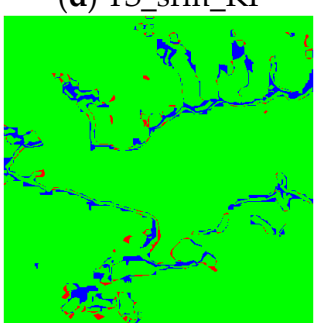

(h) TS_srm_GMM

Figure 13. The qualitative comparisons change detection results of the different algorithms over LiangZi Lake.

In the same way, several conclusions can be obtained from Figure 13. The TS_srm_GMM approach shows a better performance than the other approaches and it obtains a lower $F A$ than other approaches (such as TS_GMM_pixel, TS_GMM_object, Log_srm_GMM and TS_srm_KI) and it also obtains a lower OF than other approaches (such as Log_srm_GMM, TS_GMM_pixel, TS_GMM_object and TS_srm_TDES) in qualitative comparisons over LiangZi Lake.

The quantitative comparison of the eight detection schemes shown in Table 2 indicates that the proposed approach shows a better performance than the other approaches. The proposed approach achieves the best results on two indicators (OA (96.22\%) and Kappa (0.76)), and it obtains a lower FA $(1.56 \%)$ than the pixel-based $(2.07 \%)$ and object-based approaches. Moreover, the $O A$ of the change detection results is improved $(1.10 \%)$ and the false alarm rate reduced $(1.95 \%)$ using the proposed method, compared with some of the traditional methods. Both the qualitative and quantitative analyses demonstrate that the proposed method is effective and shows a significant improvement over the other methods. 
Table 2. Performance evaluation of the change detection over LiangZi Lake.

\begin{tabular}{ccccc}
\hline Method & $\boldsymbol{O A}$ & $\boldsymbol{F A}$ & $\boldsymbol{O F}$ & Kappa \\
\hline TS_GMM_pixel & $95.12 \%$ & $2.07 \%$ & $2.97 \%$ & 0.70 \\
TS_GMM_object & $95.41 \%$ & $1.90 \%$ & $2.74 \%$ & 0.73 \\
Log_srm_GMM & $95.52 \%$ & $1.87 \%$ & $2.60 \%$ & 0.72 \\
TS_srm_KI & $95.30 \%$ & $3.51 \%$ & $1.17 \%$ & 0.74 \\
TS_srm_TDES & $95.49 \%$ & $2.12 \%$ & $2.38 \%$ & 0.74 \\
TS_srm_kmeans & $94.29 \%$ & $4.58 \%$ & $1.12 \%$ & 0.71 \\
TS_srm_FCM & $94.22 \%$ & $4.67 \%$ & $1.10 \%$ & 0.71 \\
TS_srm_GMM & $96.22 \%$ & $1.56 \%$ & $2.20 \%$ & 0.76 \\
\hline
\end{tabular}

\section{Discussion}

The existing pixel-based unsupervised change detection methods using PolSAR images show certain deficiencies, such as not taking full advantage of the polarimetric information or being subject to the influence of noise and a high false alarm rate. Meanwhile, existing object-based unsupervised change detection methods using PolSAR images have difficulty in capturing the global property of the image, and the results are sensitive to segmentation. Furthermore, the results are often accompanied by degradation of the spatial details and fine structures. In this paper, to solve the problems of the existing unsupervised change detection methods using full PolSAR images, we have explored a novel approach to unsupervised change detection using multi-temporal Radarsat- 2 images from the city of Wuhan.

The proposed approach integrates the respective advantages of the test statistic, GSRM, and generalized GMM techniques to develop a new approach, named TS_srm_GMM, which can make full use of the polarimetric information and suppress the speckle noise. In the first step, the two filtered multi-temporal images are compared to generate a DI based on a test statistic that can make full use of the polarimetric information. The second step is aimed at reducing the speckle noise in a controlled way in order to maximize the discriminative capability between changed and unchanged classes through a segmentation strategy. The third step produces the change detection map according to a statistical clustering model based on a reformulation of the GMM. The results showed that the proposed approach achieves the best results on two indicators ( $O A$ and Kappa) and it obtains a lower $F A$ than the pixel-based and object-based approaches. Moreover, the $O A$ of the change detection results is improved and the false alarm rate is reduced using the proposed method, compared with some of the traditional methods.

The results obtained in this study are in agreement with the results of previous studies, despite the fact that the studies cannot be compared directly because of the differences in the data used. For example, Conradsen et al. [5] showed that the test statistic based on PolSAR images is much more sensitive to the differences than the test statistics based only on the backscatter coefficients, and Yang et al. [30] reported that object-based change detection can suppress the speckle noise.

The generalized GMM is derived under a non-Gaussian assumption for modeling the distributions of changed and unchanged classes. This parametric model is chosen because it is capable of better fitting the arbitrarily conditional densities of the classes in the DI after segmentation. In order to obtain a better change detection map, we automatically identify the optimal number of components $(k)$ for the GMM by the elbow method. This automatic change detection approach avoids the use of empirical methods for the selection of the number of components for the GMM. Both the qualitative and quantitative analyses of the results demonstrate that the efficiency of the proposed method is high, and that it shows a significant improvement over some of the traditional methods of change detection.

\section{Conclusions}

A novel method of automatic and unsupervised change detection using multi-temporal PolSAR images has been presented in this paper. The proposed approach is based on automatic generation of the DI using a test statistic and an object-based statistical clustering approach. Full PolSAR images 
contain both phase and amplitude information from the radar returns transmitted in two different polarizations, and more scattering information can be obtained than with single-channel SAR images. The test statistic is also a good strategy for obtaining the DI from multi-temporal PolSAR images. We use the GSRM algorithm to separate the same parts of the DI, which helps us to choose the threshold by generalized GMM. Two novel methodological contributions characterize this work, compared to the traditional unsupervised change detection techniques: (1) the influence of speckle noise in the DI is suppressed in the object-based statistical clustering approach; and (2) the optimal number of components for the GMM can be automatically selected. Regarding the first contribution, as a result of the speckle noise being suppressed, the proposed algorithm improves the $O A$ of the change detection and reduces the false alarm rate, compared with some of the traditional methods of change detection. The second contribution of estimating the optimal number of components for the GMM results in the minimum time consumption and the highest change detection accuracy. The experimental results obtained on two different multi-temporal SAR images (YanDong Lake and LiangZi Lake near the city of Wuhan, China) confirm the effectiveness of the proposed approach. Although the results obtained are very encouraging, further experiments with additional scenes should be conducted and the method should be improved. For example, the EM algorithm in the generalized GMM based on the clustering technique is prone to falling into local optima, and it can be time-consuming to arrive at the convergence criterion.

Acknowledgments: The authors would like to thank the National Natural Science Foundation of China (No. 91438203, No. 61371199, No. 41501382, No. 41601355, No. 41771377), the Hubei Provincial Natural Science Foundation (No. 2015CFB328, No. 2016CFB246), the National Basic Technology Program of Surveying and Mapping (No. 2016KJ0103), and the Technology of Target Recognition Based on GF-3 Program (No. 03-Y20A10-9001-15/16).

Author Contributions: Wensong Liu defined the research problem, proposed the methods, undertook most of the programming using MATLAB, and wrote the paper. Jie Yang gave some key advice. Jinqi Zhao gave useful advice, contributed to the paper writing, collected the data, and processed the datasets by PolSARpro and NEST software. Le Yang provided the ground reference.

Conflicts of Interest: The authors declare no conflict of interest.

\section{References}

1. Singh, A. Review Article Digital change detection techniques using remotely-sensed data. Int. J. Remote Sens. 1989, 10, 989-1003. [CrossRef]

2. Bruzzone, L.; Bovolo, F. A novel framework for the design of change-detection systems for very-high-resolution remote sensing images. Proc. IEEE 2013, 101, 609-630. [CrossRef]

3. Hachicha, S.; Chaabane, F. On the SAR change detection review and optimal decision. Int. J. Remote Sens. 2014, 35, 1693-1714. [CrossRef]

4. Giustarini, L.; Hostache, R.; Matgen, P.; Schumann, G.J.-P.; Bates, P.D.; Mason, D.C. A change detection approach to flood mapping in urban areas using TerraSAR-X. IEEE Trans. Geosci. Remote Sens. 2013, 51, 2417-2430. [CrossRef]

5. Conradsen, K.; Nielsen, A.A.; Schou, J.; Skriver, H. A test statistic in the complex wishart distribution and its application to change detection in polarimetric SAR data. IEEE Trans. Geosci. Remote Sens. 2003, 41, 4-19. [CrossRef]

6. Zhao, L.; Yang, J.; Li, P.; Zhang, L. Seasonal inundation monitoring and vegetation pattern mapping of the Erguna floodplain by means of a RADARSAT-2 fully polarimetric time series. Remote Sens. Environ. 2014, 152, 426-440. [CrossRef]

7. Zhao, J.Q.; Yang, J.; Li, P.X.; Liu, M.Y.; Shi, Y.M. An Unsupervised Change Detection Based on Test Statistic and KI from Multi-temporal and Full Polarimetric SAR Images. Int. Arch. Photogramm. Remote Sens. Spat. Inf. Sci. 2016, XLI-B7, 611-615. [CrossRef]

8. Sun, W.; Shi, L.; Yang, J.; Li, P. Building Collapse Assessment in Urban Areas Using Texture Information From Postevent SAR Data. IEEE J. Sel. Top. Appl. Earth Obs. Remote Sens. 2016, 9, 3792-3808. [CrossRef]

9. Zhao, L.; Yang, J.; Li, P.; Zhang, L.; Shi, L.; Lang, F. Damage assessment in urban areas using post-earthquake airborne PolSAR imagery. Int. J. Remote Sens. 2013, 34, 8952-8966. [CrossRef] 
10. Zhao, L.; Yang, J.; Li, P.; Zhang, L. Characteristics Analysis and Classification of Crop Harvest Patterns by Exploiting High-Frequency MultiPolarization SAR Data. IEEE J. Sel. Top. Appl. Earth Obs. Remote Sens. 2014, 7, 3773-3783. [CrossRef]

11. Rignot, E.J.; van Zyl, J.J. Change detection techniques for ERS-1 SAR data. IEEE Trans. Geosci. Remote Sens. 1993, 31, 896-906. [CrossRef]

12. Bazi, Y.; Bruzzone, L.; Melgani, F. An unsupervised approach based on the generalized Gaussian model to automatic change detection in multitemporal SAR images. IEEE Trans. Geosci. Remote Sens. 2005, 43, 874-887. [CrossRef]

13. Moser, G.; Serpico, S.B. Generalized minimum-error thresholding for unsupervised change detection from SAR amplitude imagery. IEEE Trans. Geosci. Remote Sens. 2006, 44, 2972-2982. [CrossRef]

14. Sumaiya, M.; Kumari, R.S.S. Logarithmic Mean-Based Thresholding for SAR Image Change Detection. IEEE Geosci. Remote Sens. Lett. 2016, 13, 1726-1728. [CrossRef]

15. Liu, M.; Zhang, H.; Wang, C.; Wu, F. Change detection of multilook polarimetric SAR images using heterogeneous clutter models. IEEE Trans. Geosci. Remote Sens. 2014, 52, 7483-7494.

16. Carincotte, C.; Derrode, S.; Bourennane, S. Unsupervised change detection on SAR images using fuzzy hidden Markov chains. IEEE Trans. Geosci. Remote Sens. 2006, 44, 432-441. [CrossRef]

17. Bouyahia, Z.; Benyoussef, L.; Derrode, S. Change detection in synthetic aperture Radar images with a sliding hidden Markov chain model. J. Appl. Remote Sens. 2008, 2, 513-526.

18. Inglada, J.; Mercier, G. A new statistical similarity measure for change detection in multitemporal SAR images and its extension to multiscale change analysis. IEEE Trans. Geosci. Remote Sens. 2007, 45, 1432-1445. [CrossRef]

19. Akbari, V.; Anfinsen, S.N.; Doulgeris, A.P.; Eltoft, T.; Moser, G.; Serpico, S.B. Polarimetric SAR Change Detection With the Complex Hotelling-Lawley Trace Statistic. IEEE Trans. Geosci. Remote Sens. 2016, 54, 3953-3966. [CrossRef]

20. Yousif, O.; Ban, Y. Object-Based Change Detection in Urban Areas Using Multitemporal High Resolution SAR Images with Unsupervised Thresholding Algorithms; Multitemporal Remote Sensing; Springer: Berlin, Germany, 2016; pp. 89-105.

21. Yetgin, Z. Unsupervised change detection of satellite images using local gradual descent. IEEE Trans. Geosci. Remote Sens. 2012, 50, 1919-1929. [CrossRef]

22. Bunch, J.R.; Fierro, R.D. A constant-false-alarm-rate algorithm. Linear Algebra Appl. 1992, 172, $231-241$. [CrossRef]

23. Otsu, N. A threshold selection method from gray-level histograms. Automatica 1975, 11, 23-27. [CrossRef]

24. Kapur, J.N.; Sahoo, P.K.; Wong, A.K.C. A new method for gray-level picture thresholding using the entropy of the histogram. Comput. Vis. Graph. Image Process. 1985, 29, 140. [CrossRef]

25. Kittler, J.; Illingworth, J. Minimum error thresholding. Pattern Recognit. 1986, 19, 41-47. [CrossRef]

26. Jansing, E.D.; Albert, T.A.; Chenoweth, D.L. Two-dimensional entropic segmentation. Pattern Recognit. Lett. 1999, 20, 329-336. [CrossRef]

27. Qi, Z.; Yeh, A.G.-O.; Li, X.; Zhang, X. A three-component method for timely detection of land cover changes using polarimetric SAR images. ISPRS J. Photogramm. Remote Sens. 2015, 107, 3-21. [CrossRef]

28. Rasmussen, C.E. The infinite Gaussian mixture model. Adv. Neural Inf. Proc. Syst. 2000, 10, 554-560.

29. Greenspan, H.; Ruf, A.; Goldberger, J. Constrained Gaussian mixture model framework for automatic segmentation of MR brain images. IEEE Trans. Med. Imaging 2006, 25, 1233-1245. [CrossRef] [PubMed]

30. Yang, W.; Yang, X.; Yan, T.; Song, H.; Xia, G. Region-Based Change Detection for Polarimetric SAR Images Using Wishart Mixture Models. IEEE Trans. Geosci. Remote Sens. 2016, 54, 6746-6756. [CrossRef]

31. Lang, F.; Yang, J.; Li, D.; Zhao, L.; Shi, L. Polarimetric SAR image segmentation using statistical region merging. IEEE Geosci. Remote Sens. Lett. 2014, 11, 509-513. [CrossRef]

32. Nock, R.; Nielsen, F. Statistical region merging. IEEE Trans. Pattern Anal. Mach. Intell. 2004, 26, 1452-1458. [CrossRef] [PubMed]

33. Nock, R. Fast and reliable color region merging inspired by decision tree pruning. In Proceedings of the 2001 IEEE Computer Society Conference on Computer Vision and Pattern Recognition, Kauai, HI, USA, 8-14 December 2001.

34. Celik, T.; Ma, K.-K. Multitemporal Image Change Detection Using Undecimated Discrete Wavelet Transform and Active Contours. IEEE Trans. Geosci. Remote Sens. 2011, 49, 706-716. [CrossRef] 
35. Zivkovic, Z. Improved adaptive Gaussian Mixture Model for Background Subtraction. In Proceedings of the IEEE 17th International Conference on Pattern Recognition, Cambridge, UK, 26 August 2004.

36. Dempster, A.P.; Laird, N.M.; Rubin, D.B. Maximum likelihood from incomplete data via the EM algorithm. J. R. Stat. Soc. Ser. B (Methodol.) 1977, 39, 1-38.

37. Bruzzone, L.; Prieto, D.F. A minimum-cost thresholding technique for unsupervised change detection. Int. J. Remote Sens. 2000, 21, 3539-3544. [CrossRef]

38. Ketchen, D.J., Jr.; Shook, C.L. The application of cluster analysis in strategic management research: An analysis and critique. Strateg. Manag. J. 1996, 17, 441-458. [CrossRef]

39. Goutte, C.; Toft, P.; Rostrup, E.; Nielsen, F.; Hansen, L.K. On clustering fMRI time series. Neuroimage 1999, 9 , 298-310. [CrossRef] [PubMed]

40. Pham, M.-T.; Mercier, G.; Michel, J. Change Detection between SAR Images Using a Pointwise Approach and Graph Theory. IEEE Trans. Geosci. Remote Sens. 2016, 54, 2020-2032. [CrossRef]

41. Stehman, S.V. Selecting and interpreting measures of thematic classification accuracy. Remote Sens. Environ. 1997, 62, 77-89. [CrossRef]

42. Zhao, J.; Yang, J.; Lu, Z.; Li, P.; Liu, W.; Yang, L. A Novel Method of Change Detection in Bi-Temporal PolSAR Data Using a Joint-Classification Classifier Based on a Similarity Measure. Remote Sens. 2017, 9, 846. [CrossRef]

(C) 2017 by the authors. Licensee MDPI, Basel, Switzerland. This article is an open access article distributed under the terms and conditions of the Creative Commons Attribution (CC BY) license (http:/ / creativecommons.org/licenses/by/4.0/). 